\title{
Spots, flares, accretion, and obscuration in the pre-main sequence binary DQ Tau
}

\author{
Á. Kóspál ${ }^{1,2}$, P. Ábrahám ${ }^{1}$, G. Zsidi ${ }^{1}$, K. Vida ${ }^{1}$, R. Szabóo ${ }^{1}$, A. Moór ${ }^{1}$, \\ and A. Pál ${ }^{1}$ \\ ${ }^{1}$ Konkoly Observatory, Research Centre for Astronomy and Earth Sciences, Hungarian \\ Academy of Sciences, Konkoly-Thege Miklós út 15-17, H-1121, Budapest, Hungary \\ ${ }^{2}$ Max Planck Institute for Astronomy, Königstuhl 17, D-69117 Heidelberg, Germany
}

\begin{abstract}
DQ Tau is a young low-mass spectroscopic binary, consisting of two almost equalmass stars on a 15.8 day period surrounded by a circumbinary disk. We analyzed DQ Tau's light curves obtained by Kepler K2, the Spitzer Space Telescope, and ground-based facilities. We observed variability phenomena, including rotational modulation by stellar spots, energetic stellar flares, brightening events around periastron due to increased accretion, and short dips due to temporary circumstellar obscuration. The study on DQ Tau will help in discovering and understanding the formation and evolution of other real-world examples of "Tatooine-like" systems. This is especially important because more and more evidence points to the possibility that all Sun-like stars were born in binary or multiple systems that broke up later due to dynamical interactions.
\end{abstract}

Keywords. stars: pre-main-sequence, accretion disks, circumstellar matter

Pre-main sequence stars are strongly linked with their circumstellar environment, causing variability at a wide range of wavelengths and timescales. The four main origins of photometric variability are variable accretion, rotational modulation due to hot or cold stellar spots, variable line-of-sight extinction, and stellar flares. The second mission of the Kepler spacecraft (K2) provided high precision photometry for many young stars in the Taurus star forming region in 2017 (Campaign 13), enabling us to search for young stellar variability, disentangle the different effects, and determine their relative contributions.

DQ Tau consists of two almost identical $0.6 M_{\odot}$ stars that orbit each other in an eccentric orbit with a period of 15.8 days. At periastron they approach each other within $12.5 R_{\odot}$. The binary is surrounded by a circumbinary protoplanetary disk, from which gas and dust are accreting onto the stars. The system is seen close to pole-on (Czekala et al. 2016), it displays quasi-periodic optical variability due to pulsed accretion (Tofflemire et al. 2017), and millimeter flares and elevated X-ray activity near periastron due to a combination of magnetic and dynamic effects (Getman et al. 2011, Kóspál et al. 2011).

Kepler K2 observed DQ Tau in 2017, providing an 80-day-long uninterrupted monitoring of the white-light brightness with a cadence of 1 min (Fig. 1). For a few weeks at the beginning of the K2 monitoring, we could observe DQ Tau from the ground using the $90 \mathrm{~cm}$ Schmidt telescope of Konkoly Observatory (Hungary), with BV $(\mathrm{RI})_{\mathrm{C}}$ filters. We complemented these with mid-infrared photometry at 3.6 and $4.5 \mu \mathrm{m}$ using the Spitzer Space Telescope during the final 11 days of the K2 campaign with a cadence of 20 hours.

The K2 data displays strong periodicity with $P=3.017 \pm 0.004$ days, consistently with the stellar rotational period. The phase-folded multi-filter optical data could be fitted with a model containing three spots that are $400 \mathrm{~K}$ cooler than the stellar photosphere and together cover $50 \%$ of the stellar surface (assuming that only one star is spotted).

After subtracting the rotational modulation (gray curve in Fig. 1), we searched for 
short, flare-like brightening events (fast rise and exponential decay). We identified 40 such events (highlighted with red in Fig. 1), which last for 100-200 minutes. The energy released in the flares is between $4.4 \times 10^{32}-1.2 \times 10^{35} \mathrm{erg}$, more powerful than usual for main sequence late-type stars, but typical for young low mass stars. The energy distribution of the flares suggests non-thermal emission mechanism. The flares occur randomly; we saw no correlation either with the rotational period (probably a consequence of very extended active regions that are always visible), or with the binary's orbital period (suggesting that the flares are single-star flares happening just above the stellar surface rather than between the two stars where their magnetospheres reconnect and separate).

After subtracting the rotational modulation and the flares, the remaining variability mostly consists of complex brightening events clustered around the periastrons, probably caused by increased accretion rate. This can be explained by pulsed accretion: the stars gravitationally perturb the inner edge of the circumbinary disk during each apoapsis passage and pull some material from the disk that eventually lands on the binary components. The strongest peaks reach $5 \times 10^{-8} M_{\odot} / \mathrm{yr}$, when the accretion luminosity goes up to $0.8 L_{\odot}$. The total mass accreted in each orbital cycle is typically about $1.2 \times 10^{-10} M_{\odot}$. Our Spitzer monitoring revealed that the increased accretion luminosity during the periastron events warmed up the inner disk temporarily by about $100 \mathrm{~K}$.

DQ Tau shows short dips of $<0.1$ mag in its light curve (highlighted with blue in Fig. 1), reminiscent of the well-known "dipper phenomenon" observed in many low-mass young stars, probably caused by dusty material lifted up from the inner edge of the disk. A rather small amount of mass $\left(7 \times 10^{-15} M_{\odot}\right)$ is enough to explain the dips in DQ Tau.

This project has received funding from the European Research Council (ERC) under the European Union's Horizon 2020 research and innovation programme under grant agreement No 716155 (SACCRED, PI: Á. Kóspál). The authors acknowledge the Hungarian National Research, Development and Innovation Office grants OTKA K-109276, OTKA K-113117, NKFIH K-115709, as well as Lendület LP2018-7/2018. KV is supported by the Bolyai János Research Scholarship of the Hungarian Academy of Sciences.

\section{References}

Czekala, I., Andrews, S. M., Torres, G., et al. 2016, ApJ, 818, 156

Getman, K. V., Broos, P. S., Salter, et al. 2011, ApJ, 730, 6

Kóspál, Á., Salter, D. M., Hogerheijde, et al. 2011, A\&A, 527, A96

Kóspál, Á., Ábrahám, P., Zsidi, G., et al. 2018, ApJ, 862, 44

Tofflemire, B. M., Mathieu, R. D., Ardila, D. R., et al. 2017, ApJ, 835, 8

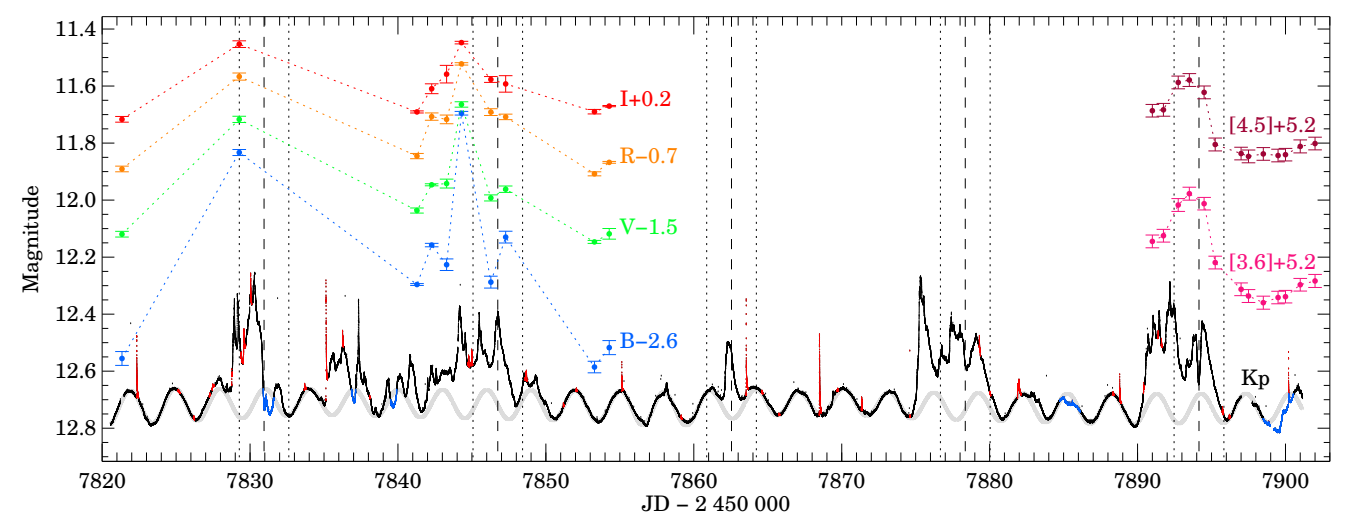

Figure 1. Light curves of DQ Tau at different wavelengths (Kóspál et al. 2018). 